\title{
Moya moya and sickle cell anemia: about 2 observations
}

\begin{abstract}
Moya's disease is a vasculopathy resulting in a progressive narrowing of the arteries of the Willis polygon responsible for ischemic or haemorrhagic stroke. At the same time: development of an abnormal capillary vascular network in "cigarette smoke" ("Moya-Moya" in Japanese). Its etiology is still poorly understood and accounts for 10 to $15 \%$ of the causes of stroke. The diagnosis of Moya Moya and the monitoring of the evolution of sickle cell anemia are currently based on MRI coupled with arteriography.

MRI and arteriography show a stenosed and occluded aspect of the arteries of the willis polygon (inner carotid artery, anterior and middle cerebral arteries) and a vascular substitution network. The objective of the study is to show the peculiarity of Moya Moya in Sickle Cell Disease through 2 observations by shedding light on the interest of MRI, MRI and arteriography affection.
\end{abstract}

Keywords: moyamoya, sickle cell disease, MRI, arteriography
Volume 4 Issue 2 - 2017

\author{
Bennani J,Aoujil F, Fikri M, Elhassani MR, \\ jiddane $M$ \\ Service of neuroradiology, Hospital of the specialties, Morocco
}

\section{Correspondence: Bennani J, Service of neuroradiology, Hospital of the specialties, Morocco,} Email jihane.bennani@gmail.com

Received: September 09, 2017 | Published: September 20, 2017

\section{Introduction}

Moya's disease is an angiogenic disease characterized by a narrowing of the distal internal carotid artery that extends to the proximal segments of the middle and anterior cerebral arteries. First described in Japan in 1955 by Takeuchi and Shimizu. The literal translation of the term moyamoya means "a foggy puff of cigarette sailing in the air" in reference to the angiographic aspect found in the disease. It is characterized by the development of these occlusions inducing the formation of supply vessels. The latter come from the parenchymatous, perforating, leptomeningeal and other transdural anastomoses. These collateral vessels have a characteristic aspect to the angiography forming a cloud of smoke: Moya Moya network. Its etiology is still poorly understood and accounts for 10 to $15 \%$ of the causes of stroke. The aim of this work is to show the peculiarity of Moya Moya in sickle cell disease through two observations.

\section{Materials and observation}

\section{Case $\mathbf{N}^{\circ} \mathbf{I}$}

This is a 27 year-old woman who has been known to have had sickle cell anemia since childhood, and who brutally presented a picture of meningeal haemorrhage caused by severe headache, vomiting. The clinical examination finds a patient subbérile subfebrile with a stiff neck. An MRI performed urgently in the three planes of space in weighted sequences T1, T2 FLAIR and angio-RM arterial. It reveals intra-ventricular haemorrhage as well as fine serpiginous vascular structures in the sylvian valleys and peri-mesencephalic. MRA shows the absence of the supraclinoid segments of the internal carotids with development of a fine cortical anastomotic network and at the Willis polygon Figure $1 \& 2$.

\section{Case $\mathbf{N}^{\circ} 2$}

This is a 16-year-old girl, followed for sickle cell anemia since the age of 2years, having an antecedent DALY at 5years of age; who presented a brutal presentation of alertness disorder with right hemicopra deficiency related to left posterior cerebral AVCI. An MRI performed urgently in the three planes of space in weighted sequences T1, T2 FLAIR and angio-RM arterial. It highlights an AVCI range of different age in the most recent is left carotid. The ARM shows the interruption of the two internal carotids at the level of their terminal segment $\mathrm{C} 1$, which are of reduced caliber in their intracavalous path with no individualization of the proximal segments of the 2 anterior and posterior cerebral arteries, a fine anastomotic network at the base "pseudomoya" Figure $3 \& 4$.
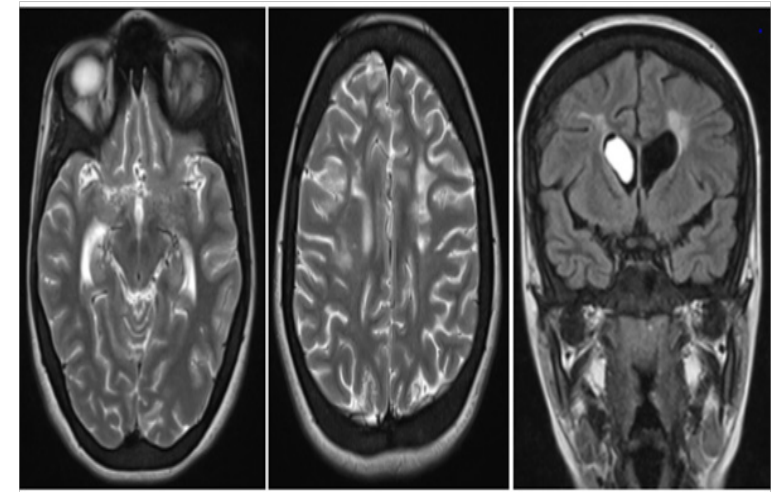

Figure I Cerebral angioplasty MRI performed urgently: in the 3 planes of space in TI, T2, FLAIR and angio-RM arterial: hyposignal T2 and hypersignal TI, intraventricular FLAIR. Demonstration of multiple serpiginous structures in hyposignal T2 and FLAIR sitting at the level of the permeesencephalic tank and the sylvian valleys. 


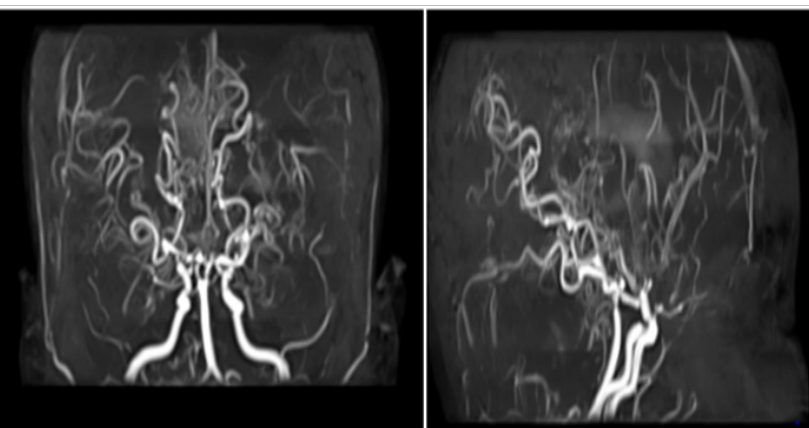

Figure 2 Arterial Angio-MRI in flight time: absence of visualization of the supraclinoid segments of the internal carotids as well as the proximal portions of the anterior and middle cerebral.A cortical anastomotic network is located at the base and visualization of the distal segments of the cerebral arteries where the flow is slowed down.

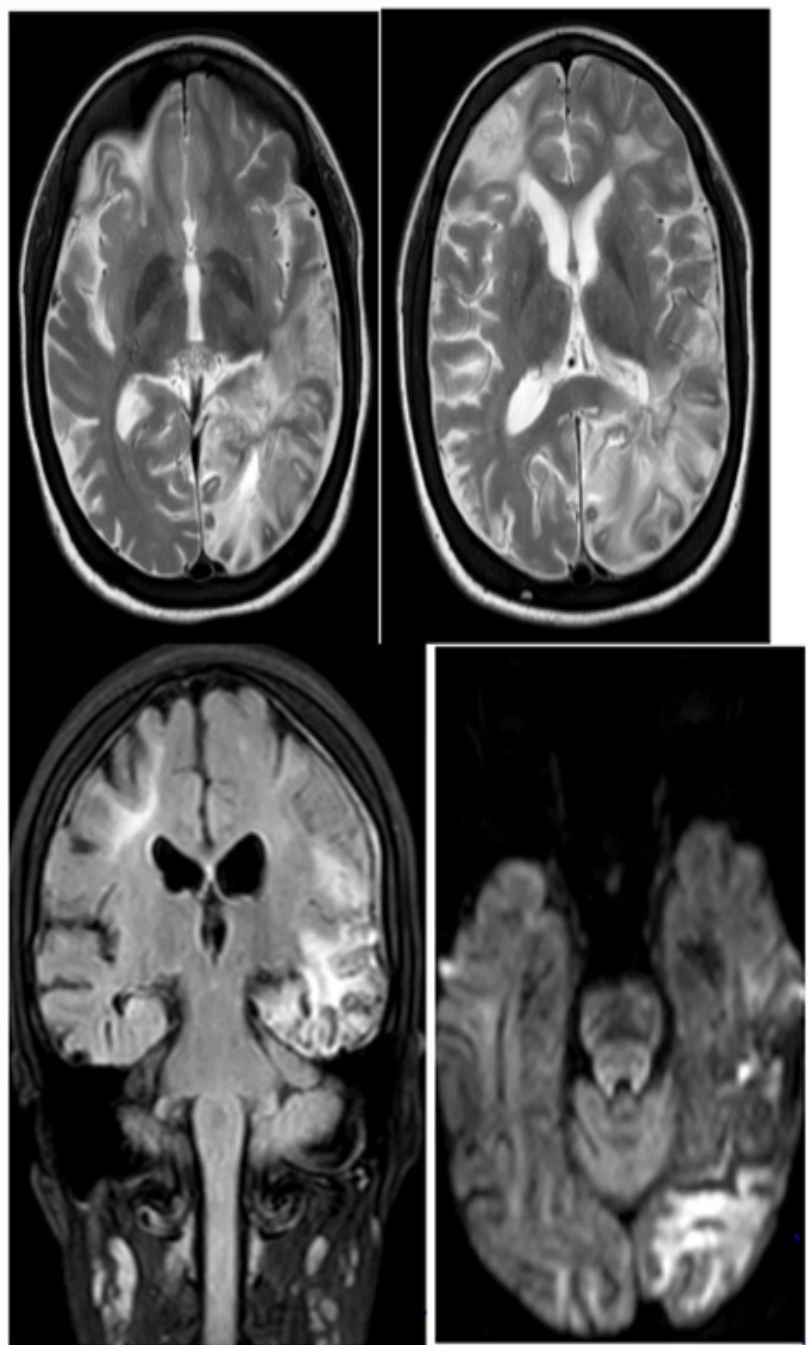

Figure 3 Angioplasty MRI performed in emergency: in the 3 planes of space in TI, T2, FLAIR and arterial angio-RM: Presence of multiple bilateral hemispherical lesional foci of cortico-subcortical topography in hyper signal T2 and FLAIR , hypo-signal diffusion in the frontal bilateral, parieto-rollodial right and associated with signal abnormalities of the white matter under cortical and gray nuclei bilaterally of chronic vascular pace.with the presence of a beach lesional temporo-parieto-occipital left in hyper signal T2, FLAIR and diffusion bI000, erasing the cortical furrows with mass effect on the occipital intersection.

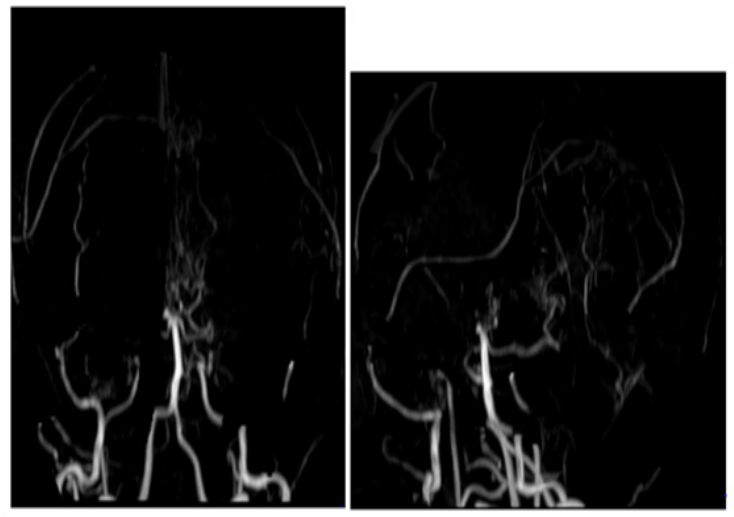

Figure 4 Arterial Angio-MRI, Interruption of the two internal carotid arteries in their $\mathrm{Cl}$ end segment, which are of reduced caliber in their intracavalous pathway with absence of individualization of the proximal segments of the 2 anterior and posterior cerebral arteries with evidence of a fine network at the "pseudomoya" base.

\section{Discussion}

Moya's disease is a chronic condition characterized by progressive occlusion of the distal portions of the supraclinoid internal carotid arteries and/or proximal portions of the middle and/or anterior cerebral arteries. As well as to the development of an irregular vascular network of supplementation realizing the aspect in "cloud of smoke", Moya Moya in Japanese. These vessels are pathological, fragile and may present dilatations and microaneurysms, resulting in the risk of rupture leading to intracerebral and/or ventricular haemorrhage. ${ }^{1}$ Moya Moya disease, which is idiopathic and isolated from Moya Moya syndrome associated with other diseases such as: Neurofibromatosis type 1, Arteriosclerosis, Trisomy21, Neoplasia, Meningitis, Radiotherapy, Sickle cell anemia.

\section{Two specific situations must be specified: association moya moya with:}

i. Radiotherapy cephalic. But the dose of radiotherapy capable of causing this effect and the interval between treatment and the occurrence of the disease varies from several months to several decades.

ii. Sickle cell disease: the affected population is underestimated. Monitoring sickle cell patients for symptoms related to cerebral ischemia is warranted.

Nowadays, sickle cell patients are exposed to vaso-occlusive seizures and chronic complications of ischemic origin that can affect virtually all organs. They occur mostly in painful bone attacks but extra-osseous manifestations may occur: acute chest syndrome, priapism, ischemic central or neurological central neurological symptoms, abdominal pain syndromes, acute multivisceral failure. Two peaks of age where the attainment of the Moya Moya is more frequent, the children around 5years and the adults around 40years. There is a female predominance: women are twice as affected as men. ${ }^{2}$

In this study, the clinical symptomatology motivating hospitalization is classic and reflects the ischemic or hemorrhagic events enamelling the course of the disease. At the end of the evolution, the importance of the motor deficits signs the severity of this disease. The delay in diagnosis is therefore frequent in young patients who are symptomatic for several months. Complementary examinations are essential for the morphological and aetiological assessment. 
The biological assessment makes it possible to isolate the Moya Moya disease itself (Nishimoto's disease), for which by definition no etiology is found. Different etiologies produce the angiographic aspect of Moya Moya disease and are grouped under the term Moya Moya syndrome. The MRI associated with MRA, a non-invasive technique, has now become a first-line examination with a sensitivity and specificity of 70 to $100 \%$. It allows the detection of parenchymal and vascular lesions. It shows signs of ischemia, haemorrhage and Moya Moya vessels. Realized in the three planes of space in weighted sequences T1, T2, FLAIR, T2*, diffusion with sequences ARM, as well as sequences $\mathrm{T} 1$ gadolinium.

The ischemic lesions will be visualized in the form of a signal T1, hyper signal T2, FLAIR and variable in diffusion according to the age, while the haemorrhagic lesions will have a variable signal in $\mathrm{T} 1$ and $\mathrm{T} 2$ according to the age, and a constant hyposignal T2* . May be subarachnoid, intra-ventricular or in the form of parenchymal hematoma. The Moya Moya vessels can be seen on the T1 sequences in the form of punctiform images in signal void, serpiginous in coronal incidence with respect to the nuclei of the base.

The ARM sequences allow the study of the polygon of willis, of the carotid bifurcations, detects the aneurysms, but its resolution is still lower than that of the digitized angiography. It does not detect microaneurysmal lesions of less than $3 \mathrm{~mm}$, may not objectify all small transdermal vessels and does not appreciate the extracranial vascular circulation necessary for surgical revascularization. Arteriography is the reference examination for diagnosis. It is essential for the assessment of the lesion. It allows to visualize intra- and extra-cranial vascularization.

Diagnosis is based on the presence of bilateral stenosis and/ or occlusion of the carotid siphons and/or proximal portions of the ACMs and ACAs.The opacification at the arterial time of a vascular network of visible bilateral supplementation around the obstructive lesions, which is the basis of the classification of Suzuki and Takaku, which classified this pathology into 6 grades of increasing gravity:

i. Grade I: stenosis of the distal portions of the internal carotid arteries.

ii. Grade II: Appearance of Moya Moya's ships.

iii. Grade III: increase in the number of vessels.

iv. Grade IV: Reduction in the number of vessels.

v. Grade V: Significant decrease in the number of vessels.

vi. Grade VI: Disappearance of the Moya Moya.

The prognosis of Moya Moya is difficult to predict because the natural history of the disease is not well known. The course of the disease can be slow with intermittent symptoms or be fulminant with rapid neurological decline. Factors influencing progression are the degree and rapidity of arterial narrowing, the patient's ability to develop collateral circulation, and the age and extent of strictures at the time of diagnosis.

If revascularization is performed before significant angiography is achieved, there is a notable improvement with a prognosis that tends to be excellent. ${ }^{3}$ Several therapeutic alternatives are discussed: therapeutic abstention, and drug therapy associated or not with surgery. Several surgical techniques have been described with the sole aim of avoiding the appearance of other ischemic lesions by increasing the collateral blood flow in the hypoperfused cortex by using most often the circulation of the external carotid artery. Direct bypass techniques (with an anastomosis between the superficial temporal artery and the middle cerebral artery (STA-MCA) or indirect (EDAS or encephaloduro-arterio-synangiogenesis) that promotes neovascularization from meningeal vessels, EMS or encephalo- myo-synangiostosis, EMAS or encephalo- myo-arterio-synangiostosis, omental transplantation).

The preventive treatment of thrombosis with antiplatelet agents, the interest of which is not formally demonstrated, remains empirical but most often proposed. Long-term studies have shown good results of surgical treatment (risk of $4 \%$ complications during the first month, $96 \%$ are safe during the next 5years). The surgical treatment of Moya Moya is effective, considered as a lasting treatment of the disease.

\section{Conclusion}

Moya Moya is a rare chronic cerebral vascular disease, idiopathic or secondary, which is a not insignificant cause of stroke in sickle cell anemia. Diagnostic delay is frequent, due to its polymorphic symptomatology. AngioMR is currently the standard imaging for initial diagnosis and surveillance. Arteriography remains a gold standard for the diagnosis and precise lesional assessment of this pathology.

\section{Acknowledgements}

None.

\section{Conflict of interest}

Author declares that there is no conflict of interest.

\section{References}

1. Edward R Smith, R Michael Scot. Moya Moya: Epidemiology, Presentation, and Diagnosis. Neurosurgery Clinics of North America. 2010;21(3):543-551

2. Ben Hassine L, Mekki N, Ben Hafdhallah J, et al. Bellagha Aspect MRI of Moya Moya in children in 5 cases - Tunis - Tunisia. Congress of the French Society of Radiology. 2012.

3. Pelissou-Guyotat V, Begey-Scherrer JC, Mamelle R. Deruty Moya Moya disease: early diagnosis and surgical treatment. About three observations. 2012. 